\title{
Cancer, clinical situation where the most between investigation and development of new molecules is made to achieve the cure of this disease
}

\author{
Adrián Pablo Hunis* \\ Director of the Career of Medical Specialist in Oncology (UBA), USA
}

\section{Introduction}

Cancer is one of the therapeutic areas that encompass older efforts in the field of research and development $(I+D)$ of new drugs globally, to the point that approximately one-third of the approval of new drugs during the year 2017 what were treatment against a tumour type as follows from the last data provided by regulatory agencies European (EMA) and American (FDA) estimates populations indicate that the number of new cases will increase $70 \%$ in decades to come reaching 24 million new cases per year in the world, in the year 2035. $40 \%$ of tumours can prevent habits healthy life: avoiding the tobacco (related to $33 \%$ of tumours), alcohol (involved in $12 \%$ of tumours), both together have an effect not sumatorio but exponential, a healthy diet, making a regular exercise and thus avoiding the sedentary and obesity (related to 1 of each 20 tumours), infections, among other carcinogens. $26 \%$ of the set of 92 positive ratings issued by the EMA in 2017 corresponded to cancer treatments in regard to the new drugs and indications, in the case of Europe $26 \%$ of the set of 92 positive ratings issued by the EMA in 2017 corresponded to cancer treatments, percentage amounting to $31 \%$ in the case of 35 drugs including new molecules. In total, 24 antitumor received the positive opinion, of which 11 are treatments including an active ingredient completely new. also, a third of orphan drugs who received approval of the regulatory agency European during the last year are new therapies against different types of tumours. these positive ratings of EMA become, in a step back in decisions authorization by the European Commission.

Thus, in the case of 35 drugs including new molecules, a total 30 have already been authorized, and they $33 \%$ are treatments cancer. the set of these new drugs, the vast majority (77\%) and are funded (included in the provision of the National health system market common European) and $30 \%$ of them corresponds with new treatments cancer. the new anti-tumour approved in the US and Europe intended to various types of tumours, as lung cancers, kidney, breast, prostate, cell carcinoma Merkel, the acute lymphoblastic leukaemia, neuroendocrine tumours and acute myeloid leukaemia, among others.

The European data coincide, in general, with the other side of the Atlantic. well, 28\% of the new drugs FDA approved US are treatments different types of cancer, to the point that one's regulatory agency considered at its annual report, which 2017 has been "another year strong for the provision of patients of new anti-tumour". the agency highlights, as well, that 2017 has been the year in which has been approved the first Gene therapy against a type of cancer, in this case a form of acute lymphoblastic leukaemia. Effort at I $+\mathrm{D}$ resulted in approval 46 new drugs by the US agency in 2017, the highest number at age 21. one-third of the new drugs have mechanisms of action other than therapies already on the market and pose a great potential improvement for health of patients.

Evolution of drugs antitumor FDA approved in the last ten years. the sustained effort has as a result the appearance of new drugs more and more effective and accurate. in the US alone approved every year, on average, thirty new drugs, although there are years in which this figure is much more.

This was the case of the last year, when approved in that country a total 46 new drugs, the highest number of the last age 21 . So, follows a report published this month by the agency of the drug US (food and drug administration, FDA) that believes that the new drugs will be used for many patients' suffering from a wide range of pathology, offering "a new hope to improve the quality of life and, in some cases, increase your chances of survival" in the case of serious illnesses.

The regulatory agency US emphasizes that one-third of the new drugs (15-46) have been classified as first-in-class, that is, as drugs mechanisms of action other than therapies already on the market and having a great potential health improvement of patients. in this group is no drugs to treat diseases such as, certain forms of multiple sclerosis, myeloid leukaemia or atopic dermatitis moderate to severe, among others. also, FDA emphasizes that 18 these new drugs (39\% of total) are indicated for the treatment of rare diseases.

In the list include, for example, new drugs for the treatment of patient's haemophilia that have developed a specific type of antibodies, or for affected of a specific form of disease Batten.

As for the rest of medications, covering a wide range of diseases as different types of lymphoma and leukaemia, Duchenne muscular dystrophy, hepatitis C., mucopolysaccharidosis, breast cancer, infection cytomegalovirus, amyotrophic lateral sclerosis, psoriasis, urinary tract infections, Parkinson's disease or cancer peritoneal, among others.

Approval by the way emergency in addition, $18 \%$ of these new drugs have been approved by a way emergency (fast track) to provide alternatives to medical needs still not covered, while 37\% have been

*Correspondence to: Adrián Pablo Hunis, Board Certified in Clinical Oncology, Assistant Professor of Internal Medicine (UBA), Director of the Career of Medical Specialist in Oncology (UBA), Professor of the Oncology Chair (U. Maimónides), USA, E-mail: aphunis@gmail.com

Received: July 09, 2018; Accepted: July 16, 2018; Published: July 18, 2018 
considered advanced therapies (breakthrough therapies) to have clinical evidence indicating that may assume a major breakthrough on treatments in effect for pathologies serious without solution.

FDA has authorized addition extension to new indications of fifteen drugs and approved before, which results in new treatment options for patients with pathologies as liver cancer (which have well with the first new therapeutic 10 years), Hodgkin's disease, multiple myeloma, myasthenia serious or Crohn's disease, among others. permission of new drugs is the result, for the most part of the intense effort in research and development undertaken by prestigious groups University where is basic research and practice medicine translational. apart, pora give an example, in the country Iberian, pharmaceutical companies invest more 1,000 million euros per year (1.085 million in 2016) at I + D of new drugs for various diseases, of which more than half (577 million euros) went to trials. according to recent data available (a June 2017) project best (today participate 45 Laboratories, 55 hospitals and four groups clinical research independent), cancer is pathology attracts the largest efforts on clinical research, to the point that $53 \%$ of all clinical trials that the pharmaceutical industry has started is developed in the area oncology.

This percentage is the highest recent years, although that oncology comes representing half of clinical investigation of the pharmaceutical industry in Europe from 2014. in the period 2010-2014, I + D of new anti-tumour supposed the $38,9 \%$ all clinical trials, percentage was slightly lower $(31,2 \%)$ in 2004-2009.

However, these new advances and drugs have been revolutionising so society most prestigious oncology worldwide, ASCO (by your stands English) American society of clinical oncology (ASCO) considered immunotherapy car $\mathrm{T}$ like the advancing most important of the year 2018. Cells T cytotoxic are a type of lymphocytes responsible for end agents' strangers to the body.

However, tumour cells are able to prevent action therapy car $\mathrm{T}$ (cell chimeric antigen receptor) modified by a Gene artificial laboratory, lymphocytes $\mathrm{T}$ cytotoxic, with of the ability to locate and attack the tumour cells. immunotherapy car $\mathrm{T}$ part of the extraction of lymphocytes T cytotoxic of each patient. These lymphocytes were adding a Gene artificial by laboratory techniques, so that is generated a receiver cell specific car (chimeric antigen receptor), for cancer cells that want to delete. Well, lymphocytes $\mathrm{T}$ pass called cells car $\mathrm{T}$.

These new cells car T equipped receptor chimeric, are now able to identify and destroy tumour cells in the patient.

American Society of Clinical Oncology (ASCO) has recognized this therapy as the progress of the year, and so it has published on your annual report.

Just need to inoculate once cells car $\mathrm{T}$ in the patient as reproduced automatically to end the disease. A new remarkable, on other treatment is that just need to inoculate once cells car T in the patient, as these new cells equipped receptor-specific tumour cells, reproduce automatically to end the disease FDA (food and drug administration).

American, approved the use of various types of this type of immunotherapy car $\mathrm{T}$. This approval has been a result of the success in front of different types of cancer, as acute lymphocytic leukaemia, the non-Hodgkin lymphoma or multiple myeloma. although immunotherapy car $\mathrm{T}$, you can result in fever sustained, low blood pressure, breathing difficulties and other symptoms Associated with the syndrome release of cytokines (CRS) that even they can compromise the patient's life, there treatments that heal the CRS in most cases. another side effects of therapy car $\mathrm{T}$ are the neurological problems as disorders speech, delirium, hallucinations or seizures, which generally referred by themselves without complication.

On the other hand, the new anti-tumour, more and more accurate and lower side effects, have had a leading role with other factors as advances in surgery and radiation therapy or efforts regarding early detection, to get best survival rates in the past decades. In fact, according to the latest estimates (2010-2017), survival relative of cancer patients stands in $53 \%$ to 5 years, a data drawing an upward trend about past periods, as in 1995-1999 the percentage stood in 49\% to five years, like the rate European, and in the period 1990-1994 in 45\% (data Europe), estimates of the database euro care.

Copyright: (C2018 Hunis AP. This is an open-access article distributed under the terms of the Creative Commons Attribution License, which permits unrestricted use, distribution, and reproduction in any medium, provided the original author and source are credited. 